Journal of Engineering and Applied Sciences 15 (7): 1687-1691, 2020

ISSN: 1816-949X

(C) Medwell Journals, 2020

\title{
Electric Load Transfer for a Solar Photovoltaic Energy System with IoT Based Alarm System
}

\author{
Jesús D. Quintero Polanco, Martin D. Bravo Obando. and Diego F. Sendoya Lozada \\ Department of Electronic Engineering, Faculty of Engineering, Surcolombiana University, \\ Neiva, Huila, Colombia
}

\begin{abstract}
A prototype automatic transfer system was designed and implemented with the purpose of initiating and encouraging the manufacture of automatic or semi-automatic equipment of this type for the electrical industry in the region. This system performs the commutation by means of two three-phase electromagnetic contactors between a photovoltaic solar energy system and the public electricity grid using a Raspberry Pi 3B card as a logical processing module, the programming of the card was carried out by means of the programming language of high level Python. Through the developer Framework7, an interface was implemented that allows the visualization of the operating parameters remotely, since, different factors of the system can be monitored voltage levels per phase, frequency per phase and the current work network from a device with iOS or Android operating system. Also, the interface generates notifications when the work network is switched, even while running in the background. The measured data are sent and linked to the interface in real time using Google's mobile platform, firebase, through a wireless internet connection, although, you also have the option of making it wired. With this prototype, we can achieve an introduction to a research and labor field little explored in our region, since, we have an option at a moderate cost, simple and with a view to interesting improvements for possible commercialization.
\end{abstract}

Key words: Electromagnetic contactors, energy, Firebase, framework 7, Python, Raspberry Pi, three-phase system

\section{INTRODUCTION}

As a result of the growth of agricultural, industrial and domestic activities, the demand for energy has increased notably, especially in emerging countries. There is a need, on the part of users, to have a permanent supply of electricity (Diaz-Rodriguez et al., 2015). Questions arise as to how to supply this energy when it is scarce or there are failures in conventional power generation sources.

Taking into account the above, renewable energy can be thought of as an alternative to those that currently provide this essential service. Renewable energies offer the possibility of generating electricity virtually without emissions, at low operating prices and in a sustainable manner. As say, Colombia is a country that has the privilege of having a characteristic geography that provides the availability of different types of alternative energies. In the specific case of photovoltaic solar energy, when implementing this type of alternatives there are valuable advantages and some limitations to be taken into account. Its advantages are its inexhaustible, renewable nature and free use of pollution. But for its use, it is necessary to take into account its intermittent nature, its variability beyond human control and its low power density. According to Ospino, in Colombia the regional geographical differences define a series of very clear factors for the incidence characteristics of solar radiation that can vary according to the geographical position (mountainous or flat) and take into account some factors such as distance to sea, average rainfall and soil conditions. This leads to differentiate the amount of solar radiation that affects each region of Colombia.

Based on the above, a photovoltaic system will not provide a constant electric fluid, since, it depends on different parameters that may be constantly changing. To solve this situation, it is proposed to use the public network which would normally be the main network but in this project, it will be a backup network for the photovoltaic solar system. It is defined in this way with the intention of maintaining a constant supply of electrical energy. How to make the change from the main network to the backup network? An automatic electric charge transfer system can be an option. The main function of load transfer is to transfer the electrical energy from a faulty power source to another backup power supply, in order to maintain the continuity of the electrical supply of the required load.

In this project a prototype of automatic transfer of electric charge was developed which is complemented

Corresponding Author: Jesús D. Quintero Polanco, Department of Electronic Engineering, Faculty of Engineering,

Surcolombiana University, Neiva, Huila, Colombia 
with a system of transmission of alarms in case of failures in the network. In this way, the owner of the system or the person in charge of the system is kept informed in real time. Additionally, it allows carrying out the connection and disconnection action between the main network (photovoltaic solar system) and/or a backup network (public network) in an autonomous way. The designed system has a visual interface where the operating parameters of the transfer, voltage and frequency levels per phase, the operation network, failure notification and network changes can be monitored.

\section{MATERIALS AND METHODS}

This project was developed in five methodological phases which are described below:

Methodological phase 1; bibliographic review: A literature review was conducted. Documents, magazines, books, theses, scientific articles and web pages related to the topics covered were consulted with the aim of having a broader knowledge base and a better projection of what was done. The technical data sheets of the devices used in the project were also reviewed to define the limitations and scope of the system based on its characteristics and operation.

Methodological phase 2; system design: A prototype automatic load transfer which meets the following characteristics was designed and implemented:

- Detection of voltage faults: absence, low and high voltage per phase

- Detection of faults by frequency: absence, low and high frequency level by phase

- Disconnection of the photovoltaic solar energy system and transfer of loads to the public network

- Reconnection of the photovoltaic solar energy system and disconnection from the public network

Currently in the region there is no manufacturing of this type of equipment. The project seeks to promote innovation in the products and services offered to its future customers, promoting industrial and commercial progress in the region.

In accordance with the requirements of the project, the design begins, defining the capabilities and scope of the transfer. The transfer must operate for three-phase power networks. It will measure the voltage and frequency of the photovoltaic system (main network) and the one supplied by the electrical distribution company (backup network).

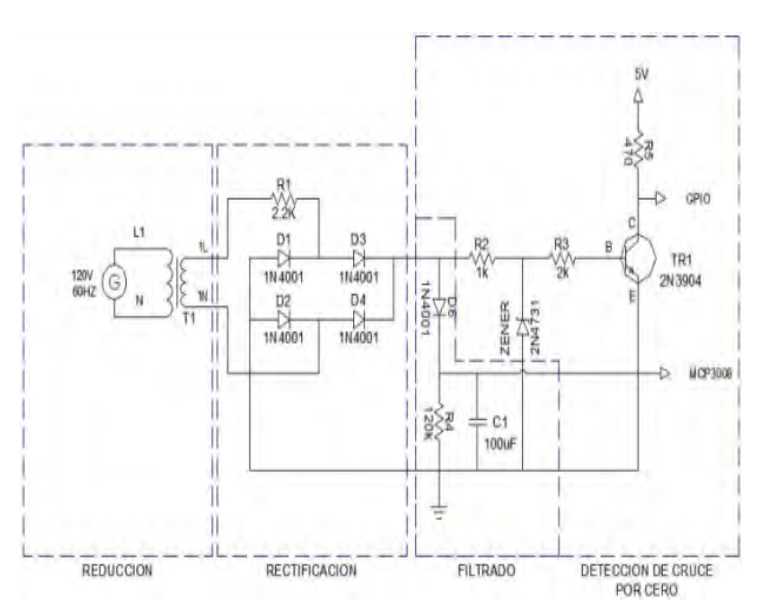

Fig. 1: Electrical diagram of signal coupling

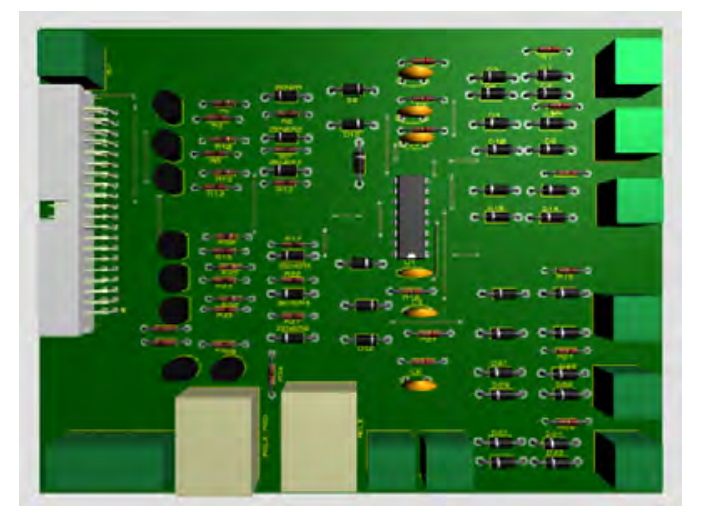

Fig. 2: PCB in Proteus ${ }^{\circledR}$

Immediately disconnect the main network system in case of phase failure: low voltage level, high voltage level, low frequency level or high frequency level. It will take the supply of the backup network, if it works correctly in the established ranges. will establish connection with the main network when it is restored. The prototype will have preference for the main network if both are available. For no reason will the load be fed simultaneously by both networks

In accordance with the established parameters, it is decided to use three-phase electromagnetic contactors CJX2 of 25 A, 120/208 V AC three-pole. As protection for the transfer, 16 A thermomagnetic breakers with a maximum voltage of $400 \mathrm{~V}$ were used.

Figure 1 and 2 shows the electrical diagram designed to perform the coupling of signals per phase. The processing that is given to the signal to perform the activation and deactivation of the contactors is described:

Frequency measurement: After reducing the amplitude of the signal with a reducing transformer, it was rectified 
with a full wave bridge. The rectified signal is introduced to a transistor that operates as a switch, generating pulses in the collector. The processing module measures the time between the pulses to calculate the frequency of the signal and in the case of obtaining an undue value makes the network change.

Voltage measurement: A similar processing was done to the frequency measurement but in this case, the digitization is done with an ADC and then it is sent to the module. In the Raspberry, an operating range is programmed for the voltage values, when the voltage of any of the phases is outside this range the contactors switch to make the network change.

After having specified how the signals and the force stage will be processed, it is fundamental to have a device that performs the control of the signals in order to centralize the operation of the transfer. Based on the above, it was decided to use the Raspberry Pi 3B board instead of an Arduino or Beagle Bone board.

To finish the design stage, the design of the PCB (Printed Circuit Board) with the Proteus ${ }^{\circledR}$ program was carried out. This is how the card is printed, where the elements of the signal processing, measurement and connection to the card stages will be located.

Methodological phase 3; information visualization: For the development of the interface, a practical and simple visualization was sought as can be seen in Fig. 3. In addition, it was sought to allow modifications and possible future improvements. This interface was developed in Framework7, this is a free and open source mobile HTML framework to develop hybrid mobile applications or web applications with a native appearance of the iOS and Android operating systems. The focus of Framework7 is to provide a development easily, clearly and with great flexibility (Framework 7, 2016).

Framework7 is compiled through a hybrid application, Cordova, an open source mobile development framework. According to Apache Software Foundation (2015), the compiler allows using standard web technologies: HTML5, CSS3 and Java Script for multiplat form development. The applications are executed within wrappers directed to each platform and depend on API (Application Programming Interface) links that comply with the standards to access the capabilities of each device such as sensors, data, network status, among others. The Apache web server is a powerful application through which critical commercial features can be delivered to customers (Krishnamurthy et al., 2008).

Methodological phase 4; real-time communication: To obtain a real-time visualization of the measured data, Firebase was used. This due to its integration with other

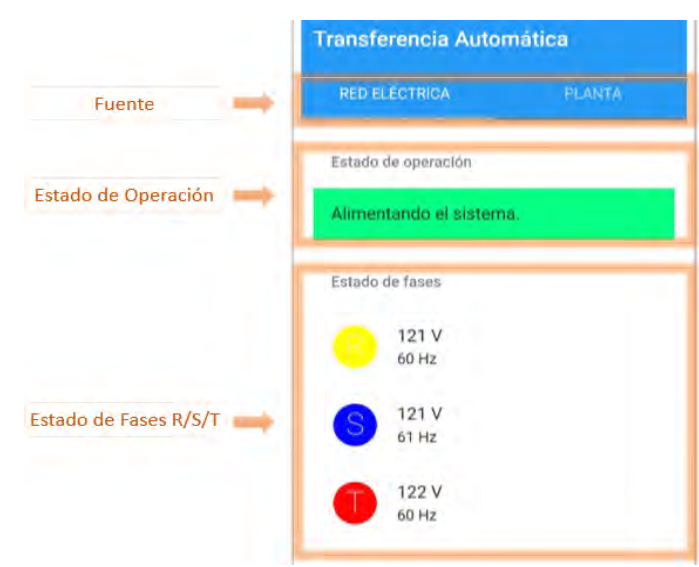

Fig. 3: Graphic interface

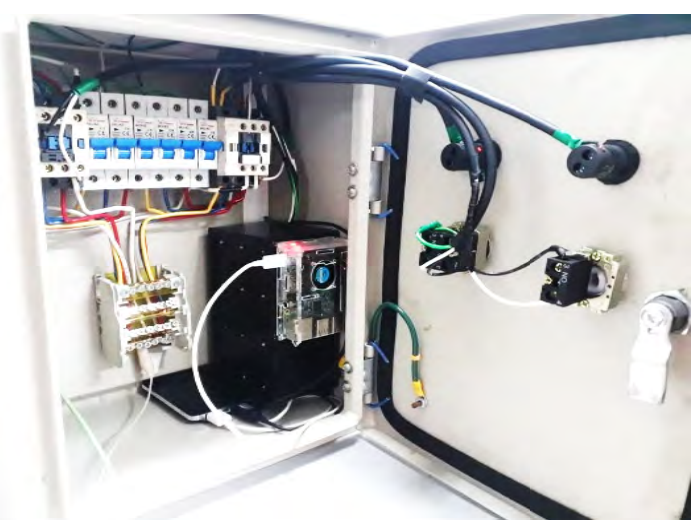

Fig. 4: Cabernetin side

platforms, since, it belongs to Google ${ }^{\circledR}$, in addition to the practicality in the programming. According to Abao et al. (2018) Firebase is a database hosted in the cloud that allows the synchronization of data between multiple users in real time. In addition, Firebase is a set of tools aimed at the creation of high-quality applications and the growth of users. With the real-time base of Firebase all the data required by the application can be saved. The data is stored in JSON format and rules can be added to allow requirements with token or only from a URL (Firebase, 2018).

Methodological phase 5; presentation of results: After making the assembly as can be seen in Fig. 4 and have the transfer in operation, the endorsement of the company, the director and the juries assigned to the project is received. As a final stage of the project, the book was written in which all the information related to the process of design, assembly and operation of the system is detailed. In addition, the working ranges, the programming algorithms used for signal processing and the algorithm of the 
designed interface are appended.

\section{RESULTS AND DISCUSSION}

The frequency and voltage measurement test was carried out with a GW Instek Model GFG-8855A signal generator with the objective of simulating the output of the transformers and achieving signal and frequency variations in amplitude. In addition, a GW Instek GDS-2062 oscilloscope is connected to observe system signals as shown in Fig. 5. In the terminals where the connection of the transformers to the circuit is made, the cables of the generator output replace it.

Different frequency values were configured, inside and outside the working range. In the same way, different signal amplitudes are adjusted in order to observe the response of the transfer. With the realization of this test, it can be shown that the resulting measurements do not have large differences, compared with those obtained under normal conditions. Next, an image that shows the performance of the test is shown, also supported by the measurements displayed in the interface.

The results obtained in the voltage and frequency measurements are compared below. Based on the tests performed and the results obtained previously, an analysis was carried out to know the existing error of the measured data. With reference to the test data contained in Table 1-3 by making an average of the values obtained in the errors of the measurements, the following is observed:

$$
\begin{gathered}
\text { Voltage error }_{\mathrm{av}}=\frac{(0.114+0.023+0.871+0.504+0.312) \mathrm{V}}{5} \\
\begin{aligned}
\text { Voltage error }_{\mathrm{av}}=\frac{1.824 \mathrm{~V}}{5} \\
\text { Voltage error }_{\mathrm{av}}=0.364 \mathrm{~V}
\end{aligned} \\
\text { Frequency error }_{\mathrm{av}}=\frac{(0.051+0.603+0.251+0.110+0.513 \mathrm{~Hz})}{5} \\
\text { Frequency error }_{\mathrm{av}}=0.305 \mathrm{~Hz}
\end{gathered}
$$

From the previous results it can be deduced that the measurements as well as in frequency and voltage have an acceptable error, although, it is greater in the voltage measurements. In addition, it can be said that there are no problems in the operation, taking into account that the operating ranges are within the range of $5 \%$ of the established nominal values.

Improvements: Taking into account that the project seeks to obtain an automatic transfer prototype, modifications and improvements are still pending in order
Table 1: Voltagemeasurements per phase

\begin{tabular}{lcc}
\hline Phase & $\begin{array}{c}\text { Voltage measured } \\
\text { by the transfer }(\mathrm{V})\end{array}$ & $\begin{array}{c}\text { Voltage measured } \\
\text { by voltmeter }(\mathrm{V})\end{array}$ \\
\hline R-network & 119 & 119.1 \\
S-network & 119 & 119.0 \\
T-network & 119 & 119.0 \\
R-photovoltaic & 119 & 119.1 \\
S-photovoltaic & 119 & 119.0 \\
T-photovoltaic & 119 & 119.1 \\
\hline
\end{tabular}

Table 2: Frequency measurements per phase

\begin{tabular}{lcc} 
Phase & $\begin{array}{c}\text { Frequency measured } \\
\text { by the transfer }(\mathrm{Hz})\end{array}$ & $\begin{array}{c}\text { Frequency measured } \\
\text { by frequency meter }(\mathrm{Hz})\end{array}$ \\
\hline R-network & 60 & 60.3 \\
S-network & 61 & 60.1 \\
T-network & 60 & 60.0 \\
R-photovoltaic & 61 & 60.1 \\
S-photovoltaic & 60 & 60.1 \\
T-photovoltaic & 60 & 60.2 \\
\hline
\end{tabular}

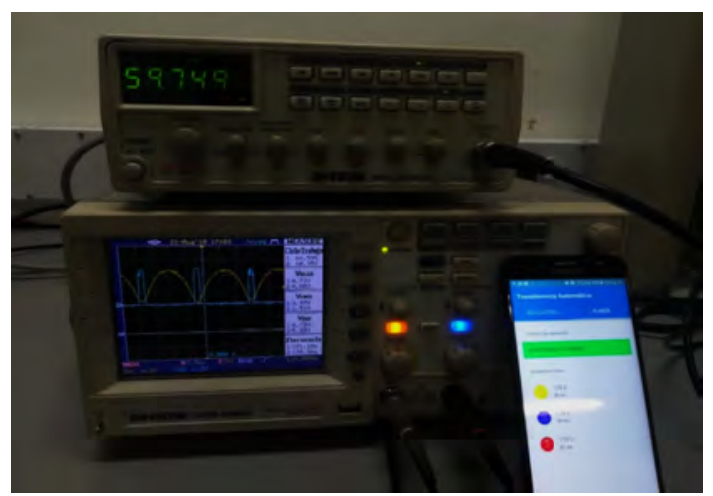

Fig. 5: Frequency and voltage tests

to obtain a more complete system for future commercialization. Below are the possible improvements. Based on the fact that residential sectors normally employ single-phase and two-phase electricity networks, equipment used in homes must have the capacity to operate with this type of network. According to the above, the transfer must extend its operating range, since, it only works in automatic mode with three-phase low voltage networks. If this improvement is made, it is recommended that a selector be added to make the choice of the type of network from the external panel, in the same way as it is done with the manual or automatic mode selector

The main objective of the implemented interface is to monitor the functioning of the system and its variables. In this way it allows only the visualization without being able to carry out any kind of control over the equipment. A possible improvement of this would clearly be the possibility of controlling variables such as the selection of operating modes and the change of work network from the mobile device.

One of the parameters of the design of the transfer is that the system is never fed with both networks simultaneously, so as not to inject power into the public 
Table 3: Relation of errors in measurements

\begin{tabular}{lcc}
\hline Generator $(\mathrm{Hz}-\mathrm{V})$ & Error in the voltage measured by the transfer $(\mathrm{V})$ & Error in the frequency measured by the transfer $(\mathrm{Hz})$ \\
\hline $30.949-120.114$ & 0.114 & -0.051 \\
$47.603-20.0230$ & 0.023 & 0.603 \\
$59.749-19.8710$ & 0.871 & 0.251 \\
$63.110-19.5040$ & 0.504 & 0.110 \\
$80.487-19.3120$ & 0.312 & -0.513 \\
\hline
\end{tabular}

network. With the new regulations related to power generation, the legal framework changes in this area. The rules, so that, users of the electric power service in the country can produce energy and sell it to the National Interconnected System were defined by the Energy and Gas Regulation Commission. This is Resolution CREG 030 of 2018 on small-scale self-generation (up to $1 \mathrm{MW}$ ) and distributed generation which defines an easy and simple mechanism for residential users of all strata as well as commercial and small industrial, to produce energy mainly to meet their own needs and can sell the surplus to the interconnected system. Based on the above, it is necessary that the transfers used with photovoltaic systems or any other type of non-conventional energy source have the capacity to inject energy into the public network to have a better use of the installed system. It is worth mentioning that in order to be self-generating energy and sell it to the National Interconnected System (SIN), in addition to the installation of electrical energy production systems, it is necessary to have a meter that can measure the energy in both directions and some protection elements of the facilities, Portfolio.

\section{CONCLUSION}

According to the design proposed for the prototype of automatic load transfer, it was possible to comply with the initially proposed requirements. A simple but robust system was obtained which allows easy on-site control and remote monitoring in real time through a visual interface with a comfortable and simple design that in turn informs its current status thanks to notifications that are sent to the configured mobile devices. The tests carried out on the system in frequency, voltage and operation measurements in general were successful, showing a great reliability of the system and its correct operation.

Although, under normal conditions the system is expected to operate in automatic mode, manual mode is also necessary in the transfer. With the inclusion of the manual operation mode, it is possible to have a status to perform different types of tests on the operation of the system and if necessary, perform corrective and preventive maintenance of the power supplies or elements of the same system.

Taking into account that the objective of the project was to design and implement a prototype, it can be said that there is a very good start to continue working on a product that can be implemented on a larger scale. This offers the possibility of making improvements and corrections to obtain a system of greater precision and better performance.

\section{REFERENCES}

Abao, R.P., C.V. Malabanan and A.P. Galido, 2018. Design and development of foodGo: A mobile application using situated analytics to augment product information. Procedia Comput. Sci., 135: 186-193.

Apache Software Foundation, 2015. Overview. Apache Software Foundation, Forest Hill, Maryland, USA.

Diaz-Rodriguez, J.L., L.D. Pabon-Fernandez and J.L. Contreras-Pena, 2015. Low-cost platform for the evaluation of single phase electromagnetic phenomena of power quality according to the IEEE 1159 standard. Dyna, 82: 119-129.

Firebase, 2018. [Firebase by platform]. Firebase, San Francisco, California, USA. (In Spanish) https://firebase.google.com/docs/?hl=es-419

Framework7, 2016. Framework7 to Introduction. GitHub, USA. https://framework7.io/docs/introduction.html

Krishnamurthy, M., E.S. Seagren, R. Alder, A.W. Bayles, J. Burke, S. Carter and E. Faskha, 2008. Apache Web Server Hardening. In: How to Cheat at Securing Linux, Krishnamurthy, M., E.S. Seagren, R. Alder, A.W. Bayles, J. Burke, S. Carter and E. Faskha (Eds.). Syngress Publishing Inc., Rockland, Massachusetts, USA., pp: 383-401. 